\title{
Magee Equation 3 predicts pathologic response to neoadjuvant systemic chemotherapy in estrogen receptor positive, HER2 negative/equivocal breast tumors
}

Daniel J Farrugia ${ }^{1,5}$, Alessandra Landmann ${ }^{1,6}$, Li Zhu ${ }^{2}$, Emilia J Diego ${ }^{1}$, Ronald R Johnson ${ }^{1}$, Marguerite Bonaventura ${ }^{1}$, Atilla Soran ${ }^{1}$, David J Dabbs ${ }^{3}$, Beth Z Clark ${ }^{3}$, Shannon L Puhalla ${ }^{4}$, Rachel C Jankowitz ${ }^{4}$, Adam M Brufsky ${ }^{4}$, Barry C Lembersky ${ }^{4}$, Gretchen M Ahrendt ${ }^{1}$, Priscilla F McAuliffe ${ }^{1}$ and Rohit Bhargava ${ }^{3}$

${ }^{1}$ Division of Surgical Oncology, Department of Surgery, University of Pittsburgh School of Medicine, Pittsburgh, PA, USA; ${ }^{2}$ Department of Biostatistics University of Pittsburgh, Pittsburgh, PA, USA; ${ }^{3}$ Division of Breast and Gynecologic Pathology, Department of Pathology, University of Pittsburgh School of Medicine, Pittsburgh, PA, USA and ${ }^{4}$ Division of Hematology/Oncology, Department of Medicine, University of Pittsburgh School of Medicine, Pittsburgh, PA, USA

\begin{abstract}
Magee Equations were derived as an inexpensive, rapid alternative to Oncotype DX. The Magee Equation 3 utilizes immunohistochemical and FISH data for estrogen receptor (ER), progesterone receptor (PR), HER2 and $\mathrm{Ki}-67$ for its calculation (24.30812+ERIHC $\times(-0.02177)+\mathrm{PRIHC} \times(-0.02884)+(0$ for HER2 negative, 1.46495 for equivocal, 12.75525 for HER2 positive)+Ki- $67 \times 0.18649)$. We hypothesize that Magee Equation 3 scores from pretherapy core biopsy can predict response to neoadjuvant systemic chemotherapy. A prospectively-maintained database of patients who received neoadjuvant systemic therapy from 2010 to 2014 at a single institution was retrospectively reviewed. Pathologic complete response was defined as absence of invasive tumor in the breast and regional lymph nodes. Of the 614 cases, tumors with missing immunohistochemical results and those that were ER negative or HER2 positive were excluded. This resulted in 237 ER positive, HER2 negative/equivocal tumors that formed the basis of this study. Magee Equation 3 scores were divided into 3 categories similar to Oncotype DX, ie, 0 to $<18$ (low), 18 to $<31$ (intermediate), and 31 or higher (high) scores. The pathologic complete response rate for low, intermediate and high Magee Equation 3 scores was $0 \%$, $4 \%$, and $36 \%$, respectively. Patients with high Magee Equation 3 scores were 13 times more likely to achieve pathologic complete response compared to those with Magee Equation 3 scores less than $31(95 \% \mathrm{Cl} 5.09-32.87$, $\boldsymbol{P}<\mathbf{0 . 0 0 0 1 )}$. For patients that did not achieve pathologic complete response, high Magee Equation 3 correlated with higher recurrence rate, with the majority occurring in patients with positive lymph nodes in the resection specimen. Magee Equation 3 score $\geq 31$ predicts pathologic complete response in the neoadjuvant setting and for tumor recurrence, when pathologic complete response is not achieved. These results show the utility of Magee Equation 3 in predicting patients who will benefit from chemotherapy but warrant prospective multiinstitutional validation.
\end{abstract}

Modern Pathology (2017) 30, 1078-1085; doi:10.1038/modpathol.2017.41; published online 26 May 2017

Correspondence: Dr R Bhargava, MD, Department of Pathology, Magee-Womens Hospital of UPMC, 300 Halket Street, Pittsburgh, PA 15213, USA.

E-mail: rbhargava@mail.magee.edu

${ }^{5}$ Current address: Centegra Comprehensive Breast Program, Centegra Health System, Crystal Lake, IL, USA

${ }^{6}$ Current address: University of Oklahoma Health Science Center, Oklahoma City, OK, USA

Received 28 February 2017; revised 27 March 2017; accepted 29 March 2017; published online 26 May 2017
The decision to recommend chemotherapy to patients with early stage breast cancer that is estrogen receptor positive (ER+) HER2 negative is challenging, as many of these tumors respond favorably to endocrine therapy alone. Clinical parameters, such as tumor size and lymph node status, are strong prognostic factors, ${ }^{1,2}$ but appear to have limited predictive value in assessing responsiveness to chemotherapy. Although tumor grade predicts 
tumor responsiveness to chemotherapy, it is not commonly used due to the perception of interobserver variability in tumor grading. ${ }^{3}$

Various multigene assays have been developed in an attempt to more accurately identify patients with $\mathrm{ER}+$ breast cancer who will benefit from chemotherapy. Oncotype DX (Genomic Health, Redwood City, CA, USA), also known as the 21 gene expression assay, is one such test. ${ }^{4}$ It is reported as a numerical recurrence score ranging from 0 to 100 and divided into low-risk $(<18)$, intermediate risk (18-30), and high-risk $(\geq 31)$ categories, and current guidelines, including National Comprehensive Cancer Network (NCCN), recommend its use in women with early stage ER+ disease. Despite the predictive and prognostic information this and other genomic tests provide, they have limitations, including cost of over $\$ 4,000$ per test, delay in treatment while awaiting results and restricted availability in settings with limited resources.

Four of the sixteen genes in the Oncotype DX assay are routinely measured at the protein level by immunohistochemistry, including ER, PR, HER2, and Ki-67. For HER2 equivocal cases on immunohistochemistry, the HER2 gene status is assessed by fluorescence in situ hybridization. Our group has previously reported that these biomarkers alone, or when combined with histologic parameters, can be incorporated into a multivariable model (known as Magee Equations; http://path.upmc.edu/online Tools/MageeEquations.html) to predict the recurrence score. ${ }^{5,6}$ This approach to estimate the recurrence score has been validated internally and externally. ${ }^{6-8}$ However, the Magee Equations have never been directly tested to predict for chemotherapy responsiveness.

Since Oncotype DX recurrence score has been shown to be positively associated with chemotherapy responsiveness in the neoadjuvant setting, ${ }^{9,10}$ we hypothesized that; similarly, the Magee Equation 3 should also predict response to chemotherapy, but in an inexpensive, rapid manner. In order to have widespread applicability, we chose to focus on the Magee Equation 3 test, since this equation requires only readily available semi-quantitative results for ER, PR, HER2, and Ki-67. ${ }^{6}$

\section{Materials and methods}

After Institutional Review Board approval, retrospective review of a prospectively maintained database was conducted of 614 patients who received neoadjuvant therapy from 2010 to 2014 at Magee-Womens Hospital of the University of Pittsburgh Medical Center. Of the 614 cases, pre-therapy ER and/or HER2 results were unknown on 27 cases, and 340 cases were ER negative or HER2+. Another 10 cases lacked semi-quantitative results for which Magee Equation 3 scores could not be calculated. After excluding these cases, $237 \mathrm{ER}+$, HER2 negative or equivocal cases were included in this study. Patients who received either neoadjuvant systemic chemotherapy $(n=191,81 \%)$ or neoadjuvant endocrine therapy $(n=46,19 \%)$ were included.

Pathologic complete response was defined as absence of invasive tumor in the breast resection specimen and regional lymph nodes. Residual in situ carcinoma was allowed. In addition to pathologic complete response, estimated tumor size/volume reduction in the breast was calculated using the following equation: Estimated percent tumor size/ volume reduction $=($ (pre-therapy clinical size —'revised' pathology size)/pre-therapy clinical size) $\times$ 100. The 'revised' pathology tumor size is calculated by multiplying the largest dimension of the gross tumor bed by the invasive tumor cellularity of the tumor bed in comparison to the pre-therapy core biopsy sample.

Pre-therapy ER, PR, HER2 and Ki-67 results were available from core biopsy pathology reports and were used to calculate Magee Equation 3 scores using the equation: 24.30812+ER H-score $\times$ $(-0.02177)+\mathrm{PR}$ H-score $\times(-0.02884)+(0$ for HER2 negative, 1.46495 for equivocal, 12.75525 for HER2 positive)+Ki-67 labeling index $\times 0.18649$ (ref. 6). Magee Equation 3 scores were divided into 3 categories similar to Oncotype DX, ie, 0 to $<18$ (low), 18 to $<31$ (intermediate), and 31 or higher scores (high). The pathologic complete response rate with respect to Magee Equation 3 categories was analyzed. Estimated tumor size/volume reduction ( $>50 \%$ or $\leq 50 \%$ ) in the breast was also analyzed with respect to Magee Equation 3 categories.

Mean follow-up time was 35 months (median 34, range 6-68 months). Kaplan-Meier survival curves for disease free survival and overall survival were analyzed with respect to Magee Equation 3 categories and $P$-values were obtained using log rank test.

Statistical analysis was performed using The $\mathrm{R}$ project for Statistical Computing (https://www.rproject.org/). For continuous variables, $P$-value was obtained from two-sided Wilcoxon rank sum test. For categorical variables, $P$-value was obtained from two-sided Fisher exact test. Patients with missing/ unknown information were excluded from the test. Confidence intervals were obtained from Wald normal approximation. Multivariable analysis for prediction of pathologic complete response was performed after including variables that were significant on univariable analysis.

\section{Results}

Patient and tumor characteristics with respect to pathologic complete response for the 237 ER+, HER2 negative/equivocal cases included in this study are shown in Table 1. Overall, pathologic complete response was achieved in 25 patients (11\%). The following factors were significantly associated with pathologic complete response: younger age, premenopausal status, smaller pre-therapy tumor size, and 
Table 1 Patient and tumor characteristics

\begin{tabular}{|c|c|c|c|c|}
\hline & Total $\mathrm{N}=237$ & $\begin{array}{c}\text { Pathologic complete response not } \\
\text { achieved } \mathrm{N}=212 ; 89 \%\end{array}$ & $\begin{array}{c}\text { Pathologic complete response } \\
\text { achieved } \mathrm{N}=25 ; 11 \%\end{array}$ & P-value ${ }^{\mathrm{a}}$ \\
\hline \multicolumn{5}{|c|}{ Continuous variables: mean (Std. dev.) } \\
\hline Age & $54.3(12.2)$ & $54.8(12.4)$ & $49.6(10.2)$ & 0.0328 \\
\hline \multirow{2}{*}{ Age at menarche } & $12.7(1.7)$ & $12.8(1.8)$ & $12.3(1.4)$ & 0.1212 \\
\hline & Unknown 38 & Unknown 32 & Unknown 6 & \\
\hline \multirow[t]{2}{*}{ Body mass index } & $29.0(7.4)$ & $28.9(7.6)$ & $29.4(5.4)$ & 0.3895 \\
\hline & Unknown 53 & Unknown 48 & Unknown 5 & \\
\hline \multirow[t]{2}{*}{ Pre-therapy tumor size } & $4.2(2.7)$ & $4.4(2.7)$ & $3.2(1.9)$ & 0.0245 \\
\hline & Unknown 22 & Unknown 19 & Unknown 3 & \\
\hline Ki-67 labeling index in \% & $41.8(28.8)$ & $37.9(27.4)$ & $75(15.6)$ & $<0.0001$ \\
\hline Estrogen receptor H-score & $204(98.5)$ & $217.4(89.9)$ & $91.8(100.2)$ & $<0.0001$ \\
\hline $\begin{array}{l}\text { Progesterone receptor } \\
\text { H-score }\end{array}$ & $110.8(104)$ & $119.3(103.9)$ & $38.6(76.8)$ & $<0.0001$ \\
\hline \multicolumn{5}{|l|}{ Categorical variables: $N(\%)$} \\
\hline \multicolumn{5}{|l|}{ Menopausal status } \\
\hline Premenopausal & $107(45 \%)$ & $89(42 \%)$ & $18(72 \%)$ & \multirow[t]{3}{*}{0.0041} \\
\hline Postmenopausal & $127(54 \%)$ & $121(57 \%)$ & $6(24 \%)$ & \\
\hline Unknown & $3(1 \%)$ & $2(1 \%)$ & $1(4 \%)$ & \\
\hline \multicolumn{5}{|l|}{ Race } \\
\hline Caucasian & $218(92 \%)$ & $195(92 \%)$ & $23(92 \%)$ & \multirow[t]{4}{*}{0.7734} \\
\hline Black & $16(7 \%)$ & $14(7 \%)$ & $2(8 \%)$ & \\
\hline Asian & $1(<1 \%)$ & $1(<1 \%)$ & 0 & \\
\hline Others & $2(<1 \%)$ & $2(<1 \%)$ & 0 & \\
\hline \multicolumn{5}{|c|}{ Pre-therapy lymph node status } \\
\hline Core biopsy positive & $88(37 \%)$ & $77(36 \%)$ & $11(44 \%)$ & \multirow[t]{3}{*}{0.7979} \\
\hline Core biopsy negative & $94(40 \%)$ & $85(40 \%)$ & $9(36 \%)$ & \\
\hline Unknown & $55(23 \%)$ & $50(24 \%)$ & $5(20 \%)$ & \\
\hline \multicolumn{5}{|l|}{ Pre-therapy nuclear grade } \\
\hline Nuclear grade 1 or 2 & $112(47 \%)$ & $110(52 \%)$ & $2(8 \%)$ & \multirow[t]{3}{*}{$<0.0001$} \\
\hline Nuclear grade 3 & $109(46 \%)$ & $87(41 \%)$ & $22(88 \%)$ & \\
\hline Unknown & $16(7 \%)$ & $15(7 \%)$ & $1(4 \%)$ & \\
\hline \multicolumn{5}{|l|}{ Histology } \\
\hline Ductal & $199(84 \%)$ & $174(82 \%)$ & $25(100 \%)$ & \multirow[t]{3}{*}{0.0862} \\
\hline Lobular & $30(13 \%)$ & $30(14 \%)$ & 0 & \\
\hline Others & $8(3 \%)$ & $8(4 \%)$ & 0 & \\
\hline \multicolumn{5}{|l|}{ Progesterone receptor status } \\
\hline Positive & $173(73 \%)$ & $164(77 \%)$ & $9(36 \%)$ & \multirow[t]{2}{*}{$<0.0001$} \\
\hline Negative & $64(27 \%)$ & $48(23 \%)$ & $16(64 \%)$ & \\
\hline \multicolumn{5}{|l|}{ HER2 status } \\
\hline Negative & $201(85 \%)$ & $180(85 \%)$ & $21(84 \%)$ & \multirow{2}{*}{1.0000} \\
\hline Equivocal & $36(15 \%)$ & $32(15 \%)$ & $4(16 \%)$ & \\
\hline
\end{tabular}

For categorical variables, $P$-value is obtained from two-sided Fisher exact test. Patients with missing/unknown information are excluded from test. ${ }^{a}$ For continuous variables, $P$-value is obtained from two-sided Wilcoxon rank sum test.

pre-therapy nuclear grade 3. These were later evaluated in multi-variable analysis. Lower ER H-score $(P<0.0001)$, lower PR H-score $(P<0.0001)$, and higher Ki-67 labeling index $(P<0.0001)$ were also significantly associated with pathologic complete response.

Among the 237 patients, the distribution of Magee Equation 3 scores was low in $65(27 \%)$, intermediate in $116(49 \%)$, and high in $56(24 \%)$. The pathologic complete response rate for low, intermediate, and high Magee Equation 3 scores was 0\%, 4\%, and 36\% respectively (Table 2). Patients with high Magee Equation 3 scores were 13 times more likely to achieve pathologic complete response compared to those with Magee Equation 3 scores < 31 (95\% CI 5.09-32.87, $P<0.0001)$. Forty-six patients $(19 \%)$ received neoadjuvant endocrine therapy alone. The Magee Equation 3 scores in this subset of patients was low in 28 cases (61\%), intermediate in 17 cases $(37 \%)$, and high in only one $(2 \%)$ case. None of these patients achieved pathologic complete response. The pathologic complete response rate in the low, intermediate and high Magee Equation 3 categories changed only slightly to $0 \%, 5 \%$, and $36 \%$ respectively, after excluding the 46 patients who received neoadjuvant endocrine therapy alone $(P<0.0001)$. For patients receiving neoadjuvant chemotherapy, agents were chosen at the discretion of treating oncologists, resulting in somewhat heterogeneous treatment regimens. Nevertheless, most patients (54\%) received adriamycin, cyclophosphamide, and taxane combination chemotherapy. There was no statistically significant difference in pathologic complete response with respect to adriamycin, cyclophosphamide, and taxane chemotherapy $(P=0.5237)$, non-adriamycin based chemotherapy 
Table 2 Magee Equation 3 score categories and outcomes

\begin{tabular}{|c|c|c|c|c|c|}
\hline \multirow{2}{*}{ Outcomes } & \multicolumn{3}{|c|}{ Magee Equation 3 score categories } & \multirow{2}{*}{$\begin{array}{l}\text { Relative Risk (Magee Equation } 3 \geq 31 \text { vs Magee Equation } \\
\qquad 3<31 \text { ) with } 95 \% \text { Confidence Interval }{ }^{\mathrm{b}}\end{array}$} & \multirow{2}{*}{ P-value } \\
\hline & $\begin{array}{l}<18 \\
\mathrm{~N}=65\end{array}$ & $\begin{array}{c}18-<31 \\
\mathrm{~N}=116\end{array}$ & $\begin{array}{l}\geq 31 \\
\mathrm{~N}=56\end{array}$ & & \\
\hline $\begin{array}{l}\text { Pathologic complete } \\
\text { response }\end{array}$ & $0(0)$ & $5(4 \%)$ & $20(36 \%)$ & $12.93(5.09-32.87)$ & $<0.0001$ \\
\hline Loco-regional; & 0 & 2 & 3 & & \\
\hline Distant; & 4 & 12 & 13 & $2.87(1.57-5.25)$ & 0.0016 \\
\hline All recurrences & $4(6 \%)$ & $14(12 \%)$ & $16(29 \%)$ & & \\
\hline Death & $1(2 \%)$ & $3(3 \%)$ & $12(21 \%)$ & $9.70(3.26-28.87)$ & $<0.0001$ \\
\hline
\end{tabular}

${ }^{\mathrm{a}} P$-value is obtained from two-sided Fisher exact test. ${ }^{\mathrm{b}}$ Confidence interval is obtained from Wald normal approximation.

$(P=1.0), \quad$ non-taxane based chemotherapy $(P=0.6087)$, or when exact chemotherapy regimen was unknown $(P=0.1399)$.

Increasing Magee Equation 3 score also correlated with an increase in the estimated tumor size/volume reduction in the breast. Average tumor size/volume reduction in low, intermediate and high Magee Equation 3 score categories was $46 \%, 59 \%$, and $77 \%$ respectively. Fifty-six percent of patients in the low/intermediate Magee Equation 3 score category showed $>50 \%$ tumor size/volume reduction, compared to $80 \%$ of the patients in the high Magee Equation 3 score category $(P=0.0024)$.

With median follow up of 34 months, there were 5 loco-regional and 29 distant recurrences. Patients with high Magee Equation 3 scores had an $~ 3$-fold increased risk in overall recurrence than those with Magee Equation 3 scores $<31$ (95\% CI 1.57-5.25, $P=0.0016$ ) (Table 2). There were a total of 16 deaths in this cohort. Survival rates for low, intermediate and high Magee Equation 3 scores were 98, 97, and $79 \%$. Patients with high Magee Equation 3 scores had an $~ 10$-fold increased risk of death compared to those with Magee Equation 3 scores < 31 (95\% CI 3.26-28.87, $P<0.0001$ ) (Table 2). Kaplan-Meier survival analysis showed worse disease free survival and overall survival for the high Magee Equation 3 category (Figure 1). However, none of the patients that achieved pathologic complete response $(n=25)$ developed tumor recurrence or died during the period of follow-up.

Among patient who did not achieve pathologic complete response $(n=212)$, the majority of tumor recurrences occurred in patients with pathologically confirmed positive lymph nodes at resection after neoadjuvant therapy. The recurrence rate in posttherapy lymph node positive patients was $20 \%$ (26/127) compared to $10 \%$ (8/84) recurrences in post-therapy lymph node negative patients $(P=0.0367$; post-treatment lymph node status was not available in 1 patient; Table 3). The tumor recurrence rate was significantly higher with Magee Equation 3 scores $\geq 31$ in both lymph node negative $(P=0.0100)$ and positive $(P<0.0001)$ subsets.

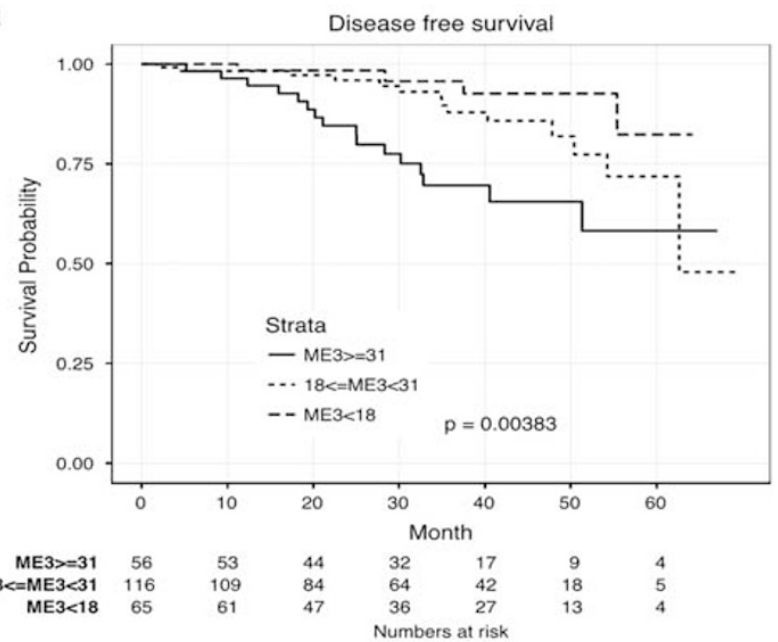

b

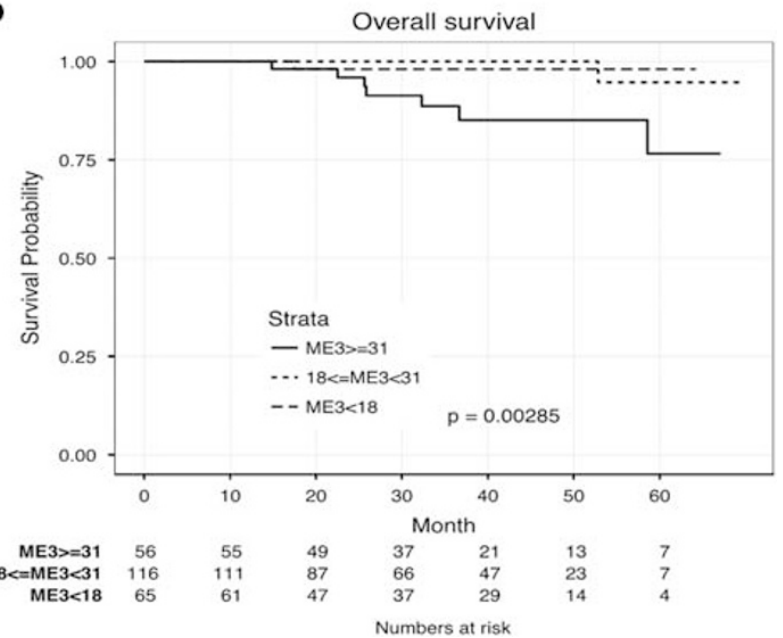

Figure 1 (a) Disease free survival Kaplan-Meier plot by Magee Equation 3 (ME3) category. (b) Overall survival Kaplan-Meier plot by Magee Equation 3 (ME3) category.

Due to significant association of pre-therapy nuclear grade 3 with pathologic complete response, we analyzed if nuclear grade provides additional information beyond that which is provided by Magee Equation 3 alone and vice versa. When cases were categorized by Magee Equation 3 categories, then 
further division by nuclear grade did not show significantly different pathologic complete response rates (Table 4). However; when cases were categorized according to nuclear grade, further divisions into Magee Equation 3 categories showed significantly different pathologic complete response rates in high grade tumors (Table 4). This is because almost all high Magee Equation 3 score cases had grade 3 nuclei, but cases with grade 3 nuclei were randomly distributed in different Magee Equation 3 categories.

A multivariable analysis of the factors significantly associated with pathologic complete response (as reported in Table 1) showed that high Magee Equation 3 was the most significant variable associated with pathologic complete response (odds ratio of 16.43, $P$ value of $<0.0001)$. Significance was also noted for pre-therapy tumor size (odds ratio of 0.73 , $P=0.0245$ ) and menopausal status (odds ratio of

Table 3 Recurrence and death rate in patients not achieving pathologic complete response $(n=212)$, categorized by lymph node status post-therapy

\begin{tabular}{|c|c|c|c|c|c|}
\hline \multirow{2}{*}{$\begin{array}{l}\text { Magee Equation } \\
3 \text { score }\end{array}$} & \multirow{2}{*}{$\mathrm{N}$} & \multicolumn{3}{|c|}{ Recurrence } & \multirow{2}{*}{ Death } \\
\hline & & $\begin{array}{l}\text { Local- } \\
\text { regional }\end{array}$ & Distant & Total & \\
\hline $\begin{array}{l}\text { Lymph node } \\
\text { negative }\end{array}$ & 84 & & & $8(10 \%)$ & 7 (8\%) \\
\hline$<18$ & 26 & 0 & 1 & $1(4 \%)$ & $0(0 \%)$ \\
\hline $18-<31$ & 40 & 0 & 2 & $2(5 \%)$ & $2(5 \%)$ \\
\hline$\geq 31$ & 18 & 1 & 4 & $5(28 \%)$ & $5(28 \%)$ \\
\hline $\begin{array}{l}\text { Lymph node } \\
\text { positive }\end{array}$ & 127 & & & $26(20 \%)$ & $9(7 \%)$ \\
\hline$<18$ & 38 & 0 & 3 & $3(8 \%)$ & $1(4 \%)$ \\
\hline $18-<31$ & 71 & 2 & 10 & $12(17 \%)$ & $1(3 \%)$ \\
\hline$\geq 31$ & 18 & 2 & 9 & $11(61 \%)$ & $7(39 \%)$ \\
\hline
\end{tabular}

Lymph node status unknown on 1 case.
0.09, $P=0.0131$ ), but not for patient age and nuclear grade.

\section{Discussion}

While there has been tremendous progress in systemic treatment of breast cancer over the last 15 years, there remains a critical need to better identify patients with ER+ tumors who will benefit from systemic chemotherapy. Tumor size is no longer the defining criterion for using chemotherapy, and even lymph node status (a highly prognostic variable) is challenged as a chemo-predictive tool, especially in post-menopausal women. ${ }^{11-13}$ Currently, multi-gene assays are used to aid chemotherapy decisions. ${ }^{14}$ However, these assays are expensive, subject to inconsistent reimbursement in the pre-operative setting, may result in a delay in initiating care, are not available in resource-poor locations and are sometimes subject to inaccurate results due to suboptimal microdissection. ${ }^{15,16}$ A readily available alternative to such multi-gene assays is Magee Equation 3, which utilizes results available in standard core-biopsy pathology reports, including semi-quantitative results for ER and PR hormone receptors, HER2, and Ki-67, to determine chemotherapy effectiveness and overall outcome.

We previously demonstrated that Magee Equation 3 can provide a fairly accurate estimate of Oncotype DX recurrence score. ${ }^{6}$ However, until now, this equation has not been systematically evaluated to determine ability to predict response to neoadjuvant treatment or outcome. Identifying tumors that will respond to chemotherapy is important in the neoadjuvant setting for overall surgical planning. There is also evidence that pathologic complete response and residual disease after chemotherapy has prognostic significance not only in triple negative and HER2 positive disease but also in ER

Table 4 Relationship between pathologic complete response rate, Magee Equation 3 score categories, and pre-therapy nuclear grade

\begin{tabular}{|c|c|c|c|c|}
\hline Categorization by & Subdivision by & $\mathrm{N}$ & Pathologic complete response N (\%) & P value \\
\hline Magee Equation 3 scores & Nuclear grade & & & \\
\hline \multirow[t]{2}{*}{ High $($ score $\geq 31$ ) } & 1 or 2 & 3 & $1(33 \%)$ & \\
\hline & 3 & 50 & $19(40 \%)$ & 1.0000 \\
\hline \multirow{2}{*}{ Intermediate (score $18-<31$ ) } & 1 or 2 & 68 & $1(1 \%)$ & \\
\hline & 3 & 44 & $3(7 \%)$ & 0.2980 \\
\hline \multirow{2}{*}{ Low (score < 18) } & 1 or 2 & 41 & $0(0 \%)$ & \\
\hline & 3 & 15 & $0(0 \%)$ & NA \\
\hline \multirow{3}{*}{$\begin{array}{l}\text { Nuclear grade } \\
1\end{array}$} & Magee Equation 3 scores & & & \\
\hline & High $($ score $\geq 31)$ & 0 & NA & \\
\hline & Intermediate or low ( score $<31$ ) & 7 & $0(0 \%)$ & NA \\
\hline \multirow[t]{2}{*}{2} & High $($ score $\geq 31$ ) & 3 & $1(33 \%)$ & \\
\hline & Intermediate or low (score < 31) & 102 & $1(1 \%)$ & 0.0566 \\
\hline \multirow[t]{2}{*}{3} & High (score $\geq 31$ ) & 50 & $19(38 \%)$ & \\
\hline & Intermediate or low (score < 31) & 59 & $3(5 \%)$ & $<0.0001$ \\
\hline
\end{tabular}

NA, not applicable.

Pre-therapy nuclear grade available on 221 cases.

${ }^{a} P$-value is obtained from two-sided Fisher exact test. 
+/HER2-negative tumors. ${ }^{17}$ Herein, we describe the clinical significance of Magee Equation 3 using our prospective institutional database of patients treated with neoadjuvant systemic treatment. We focused on Magee Equation 3 because it requires only semiquantitative results for ER, PR, HER2, and Ki-67, which are generally available on pre-therapy core biopsy reports, and may allow widespread utilization of this equation to help with decision making about systemic therapy in ER+ breast cancer.

Using only the ER+, HER2 negative/equivocal tumors from our institutional neoadjuvant database, Magee Equation 3 scores clearly identified patients that have high likelihood of pathologic complete response. A pathologic complete response rate of $36 \%$ in these ER+ patients with high Magee Equation 3 score category is similar to the pathologic complete response rate observed in triple negative tumors with first line chemotherapy. Our results using Magee Equation 3 are consistent with small number of studies analyzing Oncotype DX recurrence score for predicting pathologic complete response in ER +/HER2 negative tumors in the neoadjuvant setting. In a study of patients with HER2 negative breast cancer treated neoadjuvantly with ixabepilone and cyclophosphamide, Yardley et al showed, in a cohort of 108 patients for whom pre-therapy Oncotype DX recurrence score was available, an overall pathologic complete response rate of $26 \%$ (ie, 19 of 72 cases) in patients with high risk score and $0 \%$ (ie, 0 of 36 cases) in patients with low/intermediate risk scores. ${ }^{9}$ As expected, all ER negative patients $(n=48)$ had high risk recurrence score in this study. Of the $60 \mathrm{ER}$ + cases, pathologic complete response was identified in $17 \%$ (ie, 4 of 24 cases) of the patients with high risk recurrence scores and $0 \%$ (ie, 0 of 36 cases) in patients with low/intermediate risk scores. In an older study by Gianni et al, recurrence score was positively associated with likelihood of pathologic complete response. ${ }^{10}$ In this study, patients were treated neoadjuvantly with paclitaxel and doxorubicin, and of the 89 assessable patients, $11(12 \%)$ achieved pathologic complete response. The pathologic complete response rate in ER negative patients was $23 \%$ and in the ER+ patients was $8 \%$. Although response according to recurrence score categories was not reported, pathologic complete response was associated with higher expression of proliferation related genes, and lower expression of ER related genes, ie, the genes that determine the likelihood of high recurrence score. In another small study of 60 ER+/HER2 negative patients treated with neoadjuvant chemotherapy from our institution, Soran et al reported lack of correlation between recurrence score and tumor volume reduction in the breast. ${ }^{18}$ However, in this study, the mean recurrence score on pre-therapy core biopsy was 20 and none of the patients showed pathologic complete response, which may explain the lack of correlation to response. Nevertheless, lower ER expression level correlated with reduction in tumor volume in the breast. Comparing the studies that utilized Oncotype DX results to predict pathologic complete response to our current study using Magee Equation 3, suggests that Magee Equation 3 can perform equally well.

Although pathologic complete response after neoadjuvant therapy often results in improved long term survival, improving pathologic complete response rate using novel therapeutic agents may not always translate into increased survival. ${ }^{19-21}$ Therefore, studies with long term follow-up are valuable in understanding the significance of pathologic complete response in such circumstances. Our study did not test the efficacy of a novel therapeutic agent but rather a predictive tool to assess for pathologic complete response. Nevertheless, it is always instructive to look at long term clinical outcome. The median follow up interval of our study subjects was 34 months. All 34 recurrences, local and distant, occurred in patients that failed to achieve pathologic complete response. Recurrence and deaths were higher for patients with higher pretherapy Magee Equation 3 scores, a phenomenon similar to what is observed in triple negative tumors. ${ }^{22}$ In other words, patients with higher Magee Equation 3 scores have higher likelihood of pathologic complete response, but as group also have higher recurrence rate. This is explained by higher rate of relapse in patients that fail to achieve pathologic complete response.

Another interesting observation was the association between pre-therapy Magee Equation 3 score with post-therapy lymph node status. It is well established that residual tumor in lymph nodes after neoadjuvant therapy is a poor prognostic indicator. ${ }^{23}$ For this reason, various post-therapy prognostic indices, such as 'Residual Cancer Burden' and 'Residual Disease in Breast and Node' models, heavily weigh the amount of residual tumor within lymph nodes. ${ }^{24,25}$ Considering patients who failed to achieve pathologic complete response, a higher Magee Equation 3 score showed a higher recurrence rate within both lymph node negative and lymph node positive groups, underscoring that both biology and 'anatomy' are important in determining tumor behavior. The recurrence rate was highest $(61 \%)$ for patients with high pre-therapy Magee Equation 3 score and residual tumor in post-therapy lymph nodes (Table 3).

Our group was the first to publish a morphology and immunohistochemistry based tool to predict for Oncotype DX. ${ }^{5,6}$ Subsequently, other decision making tools have been described. ${ }^{26-30}$ These latter methods also take into account tumor grade, expression levels of PR and Ki-67 proliferation rate. However, none of the methods have been previously examined in the neoadjuvant setting with respect to pathologic complete response or to patient outcome.

The Immunohistochemistry 4 (IHC4) score described by Cuzick and colleagues ${ }^{31}$ is similar to Magee Equation 3 with some subtle differences in 
their development and use. Magee Equation 3 was formulated using a database of cases sent for clinical Oncotype DX testing (and therefore representative of the routine clinical practice), but in contrast, Immunohistochemistry 4 was developed using immunohistochemical stain scores for ER, PR, HER2, and Ki-67 on tissue blocks from patients enrolled in the Arimidex, Tamoxifen Alone or in Combination (ATAC) trial. Magee Equation 3 uses H-scores for ER and PR, but Immunohistochemistry 4 uses H-score for ER and percentage of positive cells for PR. In addition, Ki-67 labeling index need to be modified in Immunohistochemistry 4 score to be used in the equation. Immunohistochemistry 4 has been used in research studies and has been found to be comparable to Oncotype DX in providing information beyond routine clinical pathologic factors. Magee Equation 3 has been primarily used to provide an estimate of actual Oncotype DX score until now. The clinical validation provided in the current study is important and could be used to justify using Magee Equation 3 in a prospective fashion for any future neoadjuvant clinical trials.

Despite these encouraging results, our study does have some limitations. First, it is a retrospective study based on consecutive cases from routine practice, rather than from a clinical trial, which is subject to bias and heterogeneity in treatment. Nevertheless, most patients received adriamycin, cyclophosphamide, and taxane regimen, and there was no difference between the 'pathologic complete response' and 'lack of pathologic complete response' groups with respect to this regimen. It is also to be noted that follow up time in this study was relatively short, as ER positive tumors tend to recur late. Additional studies with long term follow up will be valuable to confirm the findings in the current study. Second, Magee Equation 3 requires semi-quantitative immunohistochemical results for ER, PR, HER2 and Ki-67 for which inter-observer variability can occur. The slides were not reviewed and results were taken directly from pathology reports. Some inter-observer variability is always expected, but our group has shown good inter-observer agreement with H-scores for hormone receptors. ${ }^{32}$ There are also concerns raised regarding Ki-67 labeling index reported by pathologists. ${ }^{33,34}$ Furthermore, pre-analytical factors can also interfere with accurate immunohistochemical scoring, and therefore it is important to adhere to the good laboratory practices of tissue handling and fixation. Despite these limitations, Magee Equation 3 scores were predictive of chemotherapy response and patient outcome.

In summary, Magee Equation 3 is a simple, inexpensive, accessible and highly effective tool for predicting pathologic complete response, tumor size/volume reduction and clinical outcome in ER + , HER2 negative/equivocal tumors treated with neoadjuvant chemotherapy and can be used efficiently in routine clinical practice. These results warrant prospective multi-institutional validation.

\section{Acknowledgments}

We thank all the pathologists at Magee-Womens Hospital of UPMC for meticulously reporting immunohistochemical data on breast cancers, without which this study would not have been possible.

\section{Disclosure/conflict of interest}

Authors with potential conflicts of interest are mentioned below; Emilia J Diego: DSMB for Xoft, Inc; Rachel C Jankowitz: Advisory boards for Advaxis and Biotheranostics; Shannon L Puhalla: Consulting/Advisory role for Celldex, Pfizer, Eisai, AstraZeneca, Puma, Abbvie. Research funding to institution from Abbvie, Novartis, Lilly, Pfizer, Incyte, Covance/Bayer, Puma, Roche/Genetech, AstraZeneca, Medivation; Adam M Brufsky: Consulting or Advisory Role for Pfizer,Genentech/Roche, Agendia, Celgene, Novartis, Bayer, Lilly, bioTheranostics, NanoString Technologies, Genomic Health, Puma Biotechnology; Barry C Lembersky: Speakers' Bureau for Genentech.

\section{References}

1 Cianfrocca M, Goldstein LJ. Prognostic and predictive factors in early-stage breast cancer. Oncologist 2004;9: 606-616.

2 Carter CL, Allen C, Henson DE. Relation of tumor size, lymph node status, and survival in 24,740 breast cancer cases. Cancer 1989;63:181-187.

3 Rakha EA, Reis-Filho JS, Baehner F, et al. Breast cancer prognostic classification in the molecular era: the role of histological grade. Breast Cancer Res 2010;12:207.

4 Paik S, Shak S, Tang G, et al. A multigene assay to predict recurrence of tamoxifen-treated, node-negative breast cancer. N Engl J Med 2004;351:2817-2826.

5 Flanagan MB, Dabbs DJ, Brufsky AM, et al. Histopathologic variables predict Oncotype DX recurrence score. Mod Pathol 2008;21:1255-1261.

6 Klein ME, Dabbs DJ, Shuai Y, et al. Prediction of the Oncotype DX recurrence score: use of pathologygenerated equations derived by linear regression analysis. Mod Pathol 2013;26:658-664.

7 Turner BM, Skinner KA, Tang P, et al. Use of modified Magee equations and histologic criteria to predict the Oncotype DX recurrence score. Mod Pathol 2015;28: 921-931.

8 Harowicz MR, Robinson TJ, Dinan MA, et al. Algorithms for prediction of the Oncotype DX recurrence score using clinicopathologic data: a review and comparison using an independent dataset. Breast Cancer Res Treat 2017;162:1-10.

9 Yardley DA, Peacock NW, Shastry M, et al. A phase II trial of ixabepilone and cyclophosphamide as neoadjuvant therapy for patients with HER2-negative breast cancer: correlation of pathologic complete response with the 21-gene recurrence score. Breast Cancer Res Treat 2015;154:299-308.

10 Gianni L, Zambetti M, Clark K, et al. Gene expression profiles in paraffin-embedded core biopsy tissue predict response to chemotherapy in women with 
locally advanced breast cancer. J Clin Oncol 2005;23: 7265-7277.

11 Gelber RD, Cole BF, Goldhirsch A, et al. Adjuvant chemotherapy plus tamoxifen compared with tamoxifen alone for postmenopausal breast cancer: metaanalysis of quality-adjusted survival. Lancet 1996;347: 1066-1071.

12 Schapira L, Higgins MJ. All nodes lead to chemo. Oncologist 2011;16:722-725.

13 Albain KS, Barlow WE, Shak S, et al. Prognostic and predictive value of the 21-gene recurrence score assay in postmenopausal women with node-positive, oestrogen-receptor-positive breast cancer on chemotherapy: a retrospective analysis of a randomised trial. Lancet Oncol 2010;11:55-65.

14 Harris LN, Ismaila N, McShane LM, et al. Use of biomarkers to guide decisions on adjuvant systemic therapy for women with early-stage invasive breast cancer: american society of clinical oncology clinical practice guideline. J Clin Oncol 2016;34:1134-1150.

15 Acs G, Esposito NN, Kiluk J, et al. A mitotically active, cellular tumor stroma and/or inflammatory cells associated with tumor cells may contribute to intermediate or high Oncotype DX Recurrence Scores in low-grade invasive breast carcinomas. Mod Pathol 2012;25: $556-566$.

16 Dabbs DJ, Klein ME, Mohsin SK, et al. High falsenegative rate of HER2 quantitative reverse transcription polymerase chain reaction of the Oncotype DX test: an independent quality assurance study. J Clin Oncol 2011;29:4279-4285.

17 Symmans WF, Wei C, Gould R, et al. Long-term prognostic risk after neoadjuvant chemotherapy associated with residual cancer burden and breast cancer subtype. J Clin Oncol 2017;35:1049-1060.

18 Soran A, Bhargava R, Johnson R, et al. The impact of Oncotype DX(R) recurrence score of paraffinembedded core biopsy tissues in predicting response to neoadjuvant chemotherapy in women with breas cancer. Breast Dis 2016;36:65-71.

19 Cortazar P, Zhang L, Untch M, et al. Pathological complete response and long-term clinical benefit in breast cancer: the CTNeoBC pooled analysis. Lancet 2014;384:164-172.

20 de Azambuja E, Holmes AP, Piccart-Gebhart M, et al. Lapatinib with trastuzumab for HER2-positive early breast cancer (NeoALTTO): survival outcomes of a randomised, open-label, multicentre, phase 3 trial and their association with pathological complete response. Lancet Oncol 2014;15:1137-1146.

21 Piccart-Gebhart M, Holmes E, Baselga J, et al. Adjuvant Lapatinib and Trastuzumab for Early Human Epidermal Growth Factor Receptor 2-Positive Breast Cancer: Results From the Randomized Phase III Adjuvant Lapatinib and/or Trastuzumab Treatment Optimization Trial. J Clin Oncol 2016;34:1034-1042.

22 Carey LA, Dees EC, Sawyer L, et al. The triple negative paradox: primary tumor chemosensitivity of breast cancer subtypes. Clin Cancer Res 2007;13:2329-2334.

23 Provenzano E, Bossuyt V, Viale G, et al. Standardization of pathologic evaluation and reporting of postneoadjuvant specimens in clinical trials of breast cancer: recommendations from an international working group. Mod Pathol 2015;28:1185-1201.
24 Chollet P, Abrial C, Durando X, et al. A new prognostic classification after primary chemotherapy for breast cancer: residual disease in breast and nodes (RDBN). Cancer J 2008;14:128-132.

25 Symmans WF, Peintinger F, Hatzis C, et al. Measurement of residual breast cancer burden to predict survival after neoadjuvant chemotherapy. J Clin Oncol 2007;25:4414-4422.

26 Allison KH, Kandalaft PL, Sitlani CM, et al. Routine pathologic parameters can predict Oncotype DX recurrence scores in subsets of ER positive patients: who does not always need testing? Breast Cancer Res Treat 2012;131:413-424.

27 Auerbach J, Kim M, Fineberg S. Can features evaluated in the routine pathologic assessment of lymph nodenegative estrogen receptor-positive stage I or II invasive breast cancer be used to predict the Oncotype DX recurrence score? Arch Pathol Lab Med 2010;134: 1697-1701.

28 Gage MM, Rosman M, Mylander WC, et al. A Validated Model for Identifying Patients Unlikely to Benefit From the 21-Gene Recurrence Score Assay. Clin Breast Cancer 2015;15:467-472.

29 Ingoldsby $\mathrm{H}$, Webber M, Wall D, et al. Prediction of Oncotype DX and TAILORx risk categories using histopathological and immunohistochemical markers by classification and regression tree (CART) analysis. Breast 2013;22:879-886.

30 Tang P, Wang J, Hicks DG, et al. A lower Allred score for progesterone receptor is strongly associated with a higher recurrence score of 21-gene assay in breast cancer. Cancer Invest 2010;28:978-982.

31 Cuzick J, Dowsett M, Pineda S, et al. Prognostic value of a combined estrogen receptor, progesterone receptor, Ki-67, and human epidermal growth factor receptor 2 immunohistochemical score and comparison with the Genomic Health recurrence score in early breast cancer. J Clin Oncol 2011;29:4273-4278.

32 Cohen DA, Dabbs DJ, Cooper KL, et al. Interobserver agreement among pathologists for semiquantitative hormone receptor scoring in breast carcinoma. Am J Clin Pathol 2012;138:796-802.

33 Polley MY, Leung SC, Gao D, et al. An international study to increase concordance in Ki67 scoring. Mod Pathol 2015;28:778-786.

34 Polley MY, Leung SC, McShane LM, et al. An international Ki67 reproducibility study. J Natl Cancer Inst 2013;105:1897-1906.

(c) (1) $\circledast)$ This work is licensed under a Creative Commons Attribution-NonCommercialNoDerivs 4.0 International License. The images or other third party material in this article are included in the article's Creative Commons license, unless indicated otherwise in the credit line; if the material is not included under the Creative Commons license, users will need to obtain permission from the license holder to reproduce the material. To view a copy of this license, visit http://creativecommons.org/licenses/ by-nc-nd/4.0/

(c) The Author(s) 2017 